\title{
Expression of the Receptor for Advanced Glycation End Products in Epicardial Fat: Link with Tissue Thickness and Local Insulin Resistance in Coronary Artery Disease
}

\author{
Elena Dozio, ${ }^{1}$ Elena Vianello, ${ }^{1}$ Silvia Briganti, ${ }^{2}$ John Lamont, ${ }^{3}$ Lorenza Tacchini, ${ }^{1}$ \\ Gerd Schmitz, ${ }^{4}$ and Massimiliano Marco Corsi Romanelli ${ }^{1,5}$ \\ ${ }^{1}$ Department of Biomedical Sciences for Health, University of Milan, Via L. Mangiagalli 31, 20133 Milan, Italy \\ ${ }^{2}$ Diabetology and Metabolic Disease Unit, I.R.C.C.S. Policlinico San Donato, Piazza E. Malan 1, 20097 San Donato Milanese, Italy \\ ${ }^{3}$ Randox Laboratories Ltd., R\&D, 55 Diamond Road, Crumlin, Antrim, Belfast BT29 4QY, UK \\ ${ }^{4}$ Institute for Clinical Chemistry and Laboratory Medicine, University of Regensburg, Universitätsstraße 31, \\ 93053 Regensburg, Germany \\ ${ }^{5}$ Service of Laboratory Medicine 1-Clinical Pathology, I.R.C.C.S. Policlinico San Donato, Piazza E. Malan 1, \\ 20097 San Donato Milanese, Italy
}

Correspondence should be addressed to Elena Dozio; elena.dozio@unimi.it

Received 6 June 2015; Revised 31 July 2015; Accepted 6 August 2015

Academic Editor: Vasiliki Georgiopoulou

Copyright (C) 2016 Elena Dozio et al. This is an open access article distributed under the Creative Commons Attribution License, which permits unrestricted use, distribution, and reproduction in any medium, provided the original work is properly cited.

\begin{abstract}
Increased expression of receptor for advanced glycation end products (RAGE) in adipose tissue has been associated with inflammation, adipocyte hypertrophy, and impaired insulin signal. Epicardial adipose tissue (EAT), a visceral fat surrounding the myocardium, is potentially involved in the onset/progression of coronary artery disease (CAD). To date, the role of RAGE in EAT has not been explored much. We examined whether the RAGE expression in EAT was associated with EAT adiposity and metabolic dysfunctions normally found in CAD patients. EAT samples were obtained from 33 patients undergoing open-heart surgery. EAT expression of RAGE, GLUT4, adiponenctin, GLO1, HMGB1, TLR-4, and MyD88 was analyzed by microarray. EAT thickness was quantified by echocardiography. Anthropometric measures and clinical parameters were taken. BMI, HOMA-IR, and LAP indices were calculated. With increasing RAGE expression in EAT we observed increases in EAT thickness, reduced expression of GLUT4, adiponectin, and GLO1, and elevations of HMGB1, TLR-4, and MyD88. There were significant correlations between RAGE and EAT thickness and between RAGE and the genes. LAP was higher in patients with increased RAGE expression. Our data suggest that in CAD patients RAGE may be involved in promoting EAT adiposity and metabolic dysfunction, such as impaired insulin signaling.
\end{abstract}

\section{Introduction}

The role of epicardial adipose tissue (EAT) in the onset and progression of coronary artery disease (CAD) is recognized [1], but the mechanisms and mediators promoting and linking EAT dysfunctions and CAD still need to be understood and described better.

The receptor for advanced glycation end products (RAGE) is a multiligand receptor that binds advanced glycation end products (AGE) and other endogenous nonglycated peptides, such as ligand mobility group box 1 (HMGB1), many of which are important regulators of the inflammatory process [2]. Although RAGE was initially implicated in cardiovascular complications related to diabetes [3,4], recent reports have suggested its central role in inflammation and inflammation-associated dysfunctions, such as obesity, metabolic syndrome, and atherosclerosis, even in nondiabetic conditions [5-8].

In CAD, EAT displays inflammatory features due to infiltrated macrophages and $\mathrm{T}$ cells and reduced production of protective factors in favor of detrimental proinflammatory 
mediators. This inflammatory condition may in turn contribute to the progression of atherosclerosis besides exacerbating metabolic complications in EAT $[9,10]$.

On the basis of the potential role of RAGE in adipogenesis, inflammation, and insulin resistance [5-8], we explored whether its expression in EAT was associated with EAT adiposity and the metabolic dysfunctions, such as impaired insulin signaling, normally found in CAD patients.

\section{Materials and Methods}

2.1. Study Population. Thirty-three male CAD patients undergoing coronary artery bypass grafting (CABG) surgery were enrolled in the study during their hospitalization. Exclusion criteria were acute myocardial infarction within the last month, previous or current malignant disease, major abdominal surgery within the previous 6 months, renal and liver diseases, end-stage heart failure, and more than $3 \%$ change in body weight in the previous three months. Anthropometric measures were recorded. Body mass index (BMI) was calculated by dividing the weight (in $\mathrm{Kg}$ ) by the square of the height (in meters). The study protocol, conducted in accordance with the Declaration of Helsinki, as revised in 2013, was approved by the local ethics committee (ASL Milano Due, Protocol 2516). Patients gave their written informed consent to the protocol.

2.2. Blood Collection. Blood samples were collected after overnight fasting into pyrogen-free tubes with ethylenediaminetetraacetic acid as anticoagulant. Fasting glucose, glycated hemoglobin, insulin, total and HDL cholesterol, triglycerides, and C-reactive protein (CRP) were quantified with commercial kits using Cobas 6000 analyzer (Roche Diagnostics, Milan, Italy), as previously reported [11, 12]. Precision was determined by the manufacturer using human samples and controls in an internal protocol with repeatability $(n=21)$ and intermediate precision (3 aliquots per run, 1 run per day, 21 days). Results for repeatability were glucose, $1 \%$ at $98.8 \mathrm{mg} / \mathrm{dL}$ and $0.9 \%$ at $245 \mathrm{mg} / \mathrm{dL} ; \mathrm{HbAlc}, 1.3 \%$ at $5.3 \%$ and $1.1 \%$ at $9.9 \%$; insulin, $1.9 \%$ at $6.36 \mu \mathrm{U} / \mathrm{mL}$ and $1.9 \%$ at $20.9 \mu \mathrm{U} / \mathrm{mL}$; total cholesterol, $1.1 \%$ at $88.5 \mathrm{mg} / \mathrm{dL}$ and $0.9 \%$ at $183 \mathrm{mg} / \mathrm{dL} ; \mathrm{HDL}, 0.4 \%$ at $53.4 \mathrm{mg} / \mathrm{dL}$ and $1 \%$ at $34.4 \mathrm{mg} / \mathrm{dL}$; triglycerides, $0.9 \%$ at $125 \mathrm{mg} / \mathrm{dL}$ and $0.8 \%$ at $212 \mathrm{mg} / \mathrm{dL}$; and CRP, $1.2 \%$ at $3.35 \mathrm{mg} / \mathrm{L}$ and $1.3 \%$ at $44.4 \mathrm{mg} / \mathrm{L}$.

Results for intermediate precision were glucose, $1.3 \%$ at $96.9 \mathrm{mg} / \mathrm{dL}$ and $1.1 \%$ at $241 \mathrm{mg} / \mathrm{dL} ; \mathrm{HbAlc}, 1.4 \%$ at $5.3 \%$ and $1.5 \%$ at $9.9 \%$; insulin, $2.6 \%$ at $6.36 \mu \mathrm{U} / \mathrm{mL}$ and $2.8 \%$ at $20.9 \mu \mathrm{U} / \mathrm{mL}$; total cholesterol, $1.6 \%$ at $89.3 \mathrm{mg} / \mathrm{dL}$ and $1.6 \%$ at $188 \mathrm{mg} / \mathrm{dL}$; HDL, $0.9 \%$ at $51.8 \mathrm{mg} / \mathrm{dL}$ and $1.5 \%$ at $34 \mathrm{mg} / \mathrm{dL}$; triglycerides, $2 \%$ at $123 \mathrm{mg} / \mathrm{dL}$ and $1.6 \%$ at $206 \mathrm{mg} / \mathrm{dL}$; and CRP, $2.9 \%$ at $29.1 \mathrm{mg} / \mathrm{L}$ and $1.9 \%$ at $43.6 \mathrm{mg} / \mathrm{L}$.

LDL cholesterol was calculated with the Friedewald formula. Insulin resistance index (HOMA-IR) was calculated as follows: HOMA-IR $=$ fasting insulin $[\mu \mathrm{U} / \mathrm{mL}] \times$ fasting glucose $[\mathrm{mmol} / \mathrm{L}] / 22.5$. The formula used for the lipid accumulation product (LAP) was (waist circumference [WC, $\mathrm{cm}$ ] 65) $\times($ triglycerides $[\mathrm{TG}, \mathrm{mmol} / \mathrm{L}])$.
2.3. Quantification of EAT. EAT quantification by echocardiography was performed in addition to the routine clinical examinations just before CABG surgery, usually one or two days before. Patients were examined by echocardiography using an M-mode color-Doppler VSF (Vingmed-System Five; General Electric, Horten, Norway) with a $2.5-3.5 \mathrm{MHz}$ transducer probe. EAT thickness was measured as previously reported [13].

2.4. EAT Collection. EAT biopsy samples were harvested adjacent to the proximal right coronary artery prior to starting cardiopulmonary bypass pumping. Samples were stored in Allprotect Tissue Reagent (Qiagen, Hilden, Germany) at $-20^{\circ} \mathrm{C}$ until RNA extraction.

2.5. RNA Extraction and Gene Expression Analysis. Total RNA was extracted from tissue with the RNeasy Lipid Tissue Kit according to the manufacturer's procedures (Qiagen). RNA concentration was quantified by NanoDrop 2000 (Thermo Scientific, Wilmington, Germany) and RNA integrity was assessed using the Agilent RNA 6000 Nano Kit and the Agilent 2100 Bioanalyzer (Agilent Technologies, Santa Clara, CA). Gene expression was analyzed with a onecolor microarray platform (Agilent): $50 \mathrm{ng}$ of total RNA was labeled with $\mathrm{Cy} 3$ using the Agilent Low Input Quick-Amp Labeling Kit-1 color, according to the manufacturer's directions. cRNA was purified with the RNeasy Mini Kit (Qiagen) and the amount and labeling efficiency were measured with NanoDrop. Hybridization was done using the Agilent Gene Expression Hybridization Kit and scanning with the Agilent G2565CA Microarray Scanner System.

Data were processed using Agilent Feature Extraction Software (10.7) with the single-color gene expression protocol and raw data were analyzed with ChipInspector Software (Genomatix, Munich, Germany). In brief, raw data were normalized on a single-probe level based on the array mean intensities and statistics were calculated using the SAM algorithm by Tusher et al. [14]. Changes were determined from normalized data.

2.6. Statistical Analysis. Data are expressed as mean \pm SD or number and percentage. The normality of data distribution was assessed by the Kolmogorov-Smirnoff test. Quantitative variables were compared using Student's unpaired $t$-test and Mann-Whitney and Kruskal-Wallis tests, as appropriate. The $\chi^{2}$ test was used for categorical variables. Relations between parameters were examined by the Spearman correlation test. Data were analyzed using GraphPad Prism 5.0 biochemical statistical package (GraphPad Software, San Diego, CA). A p value $<0.05$ was considered significant.

\section{Results}

3.1. Patients. Demographic, anthropometric, and clinical characteristics of the patients are shown in Table 1. Patients were classified into two groups (Q1 and Q2) according to the median value of RAGE expression in EAT (168.33 arbitrary 
TABLE 1: Demographic, anthropometric, and biochemical characteristics of coronary artery disease patients included in the study before and after classification according to the median value of RAGE expression at EAT level (Q1 and Q2 groups).

\begin{tabular}{|c|c|c|c|c|c|c|}
\hline & Median & 25th-75th percentiles & Range & $\mathrm{Q} 1($ mean $\pm \mathrm{SD})$ & $\mathrm{Q} 2($ mean $\pm \mathrm{SD})$ & $p$ \\
\hline Age (years) & 68.00 & $57.50-71.70$ & $50.00-86.00$ & $68.18 \pm 2.99$ & $66.19 \pm 2.09$ & 0.67 \\
\hline Weight (Kg) & 75.00 & $65.75-81.50$ & $52.00-135.00$ & $72.88 \pm 2.84$ & $84.17 \pm 6.12$ & 0.33 \\
\hline $\operatorname{BMI}\left(\mathrm{kg} / \mathrm{m}^{2}\right)$ & 26.20 & $23.76-27.90$ & $19.12-41.80$ & $25.14 \pm 0.71$ & $28.62 \pm 1.44$ & 0.08 \\
\hline Waist $(\mathrm{cm})$ & 102.00 & $93.50-109.05$ & $72.00-144.00$ & $95.94 \pm 3.25$ & $109.60 \pm 3.61$ & $<0.01$ \\
\hline EAT thickness (mm) & 7.50 & $5.78-8.00$ & $3.00-10.00$ & $5.50 \pm 0.67$ & $7.58 \pm 0.44$ & $<0.05$ \\
\hline Fasting glucose (mg/dl) & 82.50 & $77.50-104.50$ & $64.00-177.00$ & $99.24 \pm 7.83$ & $91.53 \pm 6.59$ & 0.79 \\
\hline Fasting insulin $(\mu \mathrm{U} / \mathrm{ml})$ & 7.11 & $4.17-10.93$ & $3.19-45.06$ & $6.97 \pm 0.71$ & $9.07 \pm 1.90$ & 0.53 \\
\hline $\operatorname{HbAlC}(\%)$ & 4.68 & $3.53-5.52$ & $2.79-7.10$ & $4.76 \pm 0.36$ & $4.58 \pm 0.30$ & 0.71 \\
\hline Total cholesterol (mg/dl) & 153.00 & $138.00-180.30$ & $88.00-261.00$ & $169.80 \pm 10.20$ & $146.40 \pm 6.81$ & 0.08 \\
\hline HDL cholesterol (mg/dl) & 44.00 & $34.25-49.25$ & $23.00-69.00$ & $44.13 \pm 2.50$ & $41.73 \pm 3.53$ & 0.58 \\
\hline LDL cholesterol (mg/dl) & 83.60 & $71.50-109.30$ & $17.60-192.00$ & $104.40 \pm 9.89$ & $82.57 \pm 4.45$ & 0.07 \\
\hline Triglycerides (mg/dl) & 107.00 & $88.50-143.00$ & $64.00-244.00$ & $114.60 \pm 9.43$ & $130.10 \pm 15.57$ & 0.40 \\
\hline CRP (mg/dl) & 0.20 & $0.10-0.95$ & $0.00-7.90$ & $0.76 \pm 0.37$ & $1.23 \pm 0.54$ & 0.44 \\
\hline Systolic blood pressure (mmHg) & 130.00 & $120.00-140.00$ & $110.00-150.00$ & $129.10 \pm 2.51$ & $131.30 \pm 3.26$ & 0.61 \\
\hline \multirow[t]{2}{*}{ Diastolic blood pressure (mmHg) } & 70.00 & $70.00-80.00$ & $60.00-80.00$ & $74.55 \pm 1.58$ & $83.00 \pm 1.49$ & 0.10 \\
\hline & $n$ & $\%$ & & $\mathrm{Q} 1(n, \%)$ & $\mathrm{Q} 2(n, \%)$ & $p$ \\
\hline Smokers & 14 & 42.42 & & $6,42.85$ & $8,57.15$ & 0.73 \\
\hline Diabetes mellitus & 9 & 27.27 & & $4,44.44$ & $5,55.56$ & 0.70 \\
\hline Hypertension & 24 & 72.73 & & $11,45.83$ & $13,54.17$ & 0.44 \\
\hline Dyslipidemia & 20 & 60.61 & & $7,35.00$ & $13,65.00$ & $<0.05$ \\
\hline Aspirin & 19 & 57.58 & & $6,31.58$ & $13,68.42$ & $<0.05$ \\
\hline Antidiabetics & 6 & 18.18 & & $3,50.00$ & $3,50.00$ & 1 \\
\hline ACEI/ARB & 24 & 72.73 & & $10,41.67$ & $14,58.33$ & 0.12 \\
\hline$\beta$-Blockers & 16 & 48.48 & & $7,43.75$ & $9,56.25$ & 0.49 \\
\hline Calcium channel blockers & 6 & 18.18 & & $3,50.00$ & $3,50.00$ & 1 \\
\hline Statins & 22 & 66.67 & & $8,36.36$ & $14,63.64$ & $<0.05$ \\
\hline
\end{tabular}

ACEI: angiotensinogen-converting enzyme inhibitor; ARB: angiotensin receptor blockade; BMI: body mass index; CRP: C-reactive protein; EAT: epicardial adipose tissue; HbA1C: glycated hemoglobin. Data are expressed as median, 25th-75th percentiles and range or number $(n)$, and $\%$ or mean \pm standard deviation (SD).

unit, A.U.). Patients were 17 in Q1 and 16 in Q2 and the mean value of RAGE expression was 140.29 A.U. in Q1 and 233.33 A.U. in Q2 ( $p<0.001)$. No statistically significant differences were observed in clinical parameters between the two groups. There were higher percentages of dyslipidemic patients and more patients taking statin and aspirin in the Q2 group $(p<0.05$ for all).

3.2. Increased RAGE Expression in EAT Is Associated with Greater Echocardiographic EAT Thickness and Higher Adiposity Indices. Echocardiographic EAT thickness and WC, a marker of visceral fat distribution, were higher in group Q2 than Q1 ( $p<0.05$ and $p<0.01$, resp.) (Figure $1(\mathrm{a})$ and Table 1). Positive correlations were seen between RAGE-EAT thickness $(r=0.48, p<0.05)$ and RAGE-WC $(r=0.35, p<$ 0.05 ) (Figure 1(b)). The relation between increased RAGE expression and greater EAT thickness was confirmed by classifying patients according to the median EAT thickness $(7.5 \mathrm{~mm})$. RAGE expression in the upper group (EAT thickness $>7.5 \mathrm{~mm})$ was about 1.3 times higher $(p<0.05)$ than in the lower group (EAT thickness $<7.5 \mathrm{~mm}$ ) (Figure 1(c)).
3.3. Increased RAGE Expression in EAT Is Associated with Lower GLUT4 and Adiponectin Expression and a Higher LAP Index. Patients in the upper group of RAGE expression in EAT (Q2) had about 1.6 times lower levels of both the insulinsensitizing adipokine adiponectin and the insulin-responsive glucose transporter 4 (GLUT4) than in the Q1 group ( $p<$ 0.05 for both) (Figure 2). There was an inverse correlation between RAGE-adiponectin $(r=-0.40, p<0.05)$ and RAGE-GLUT4 $(r=-0.60, p<0.001)$.

Examining the relation between RAGE expression in EAT and LAP and HOMA-IR, two parameters of insulin resistance, only LAP was higher in Q2 than in Q1 (Figure 2).

3.4. Increased RAGE Expression in EAT Is Associated with Lower Expression of the Antioxidant Glyoxalase 1 System and Higher HMGB1, TLR-4, and MyD88. Patients in the Q2 group of RAGE expression in EAT had about a 1.3 times lower level of glyoxalase 1 (GLO1), a system playing a critical role in the prevention of glycation reactions mediated by methylglyoxal, glyoxal, and other RAGE ligands, and about 1.2 times the level of the endogenous RAGE ligand HMGB1 compared to group Q1 $(p<0.01$ for both) (Figure 3(a)). Both TLR-4 and MyD88, 

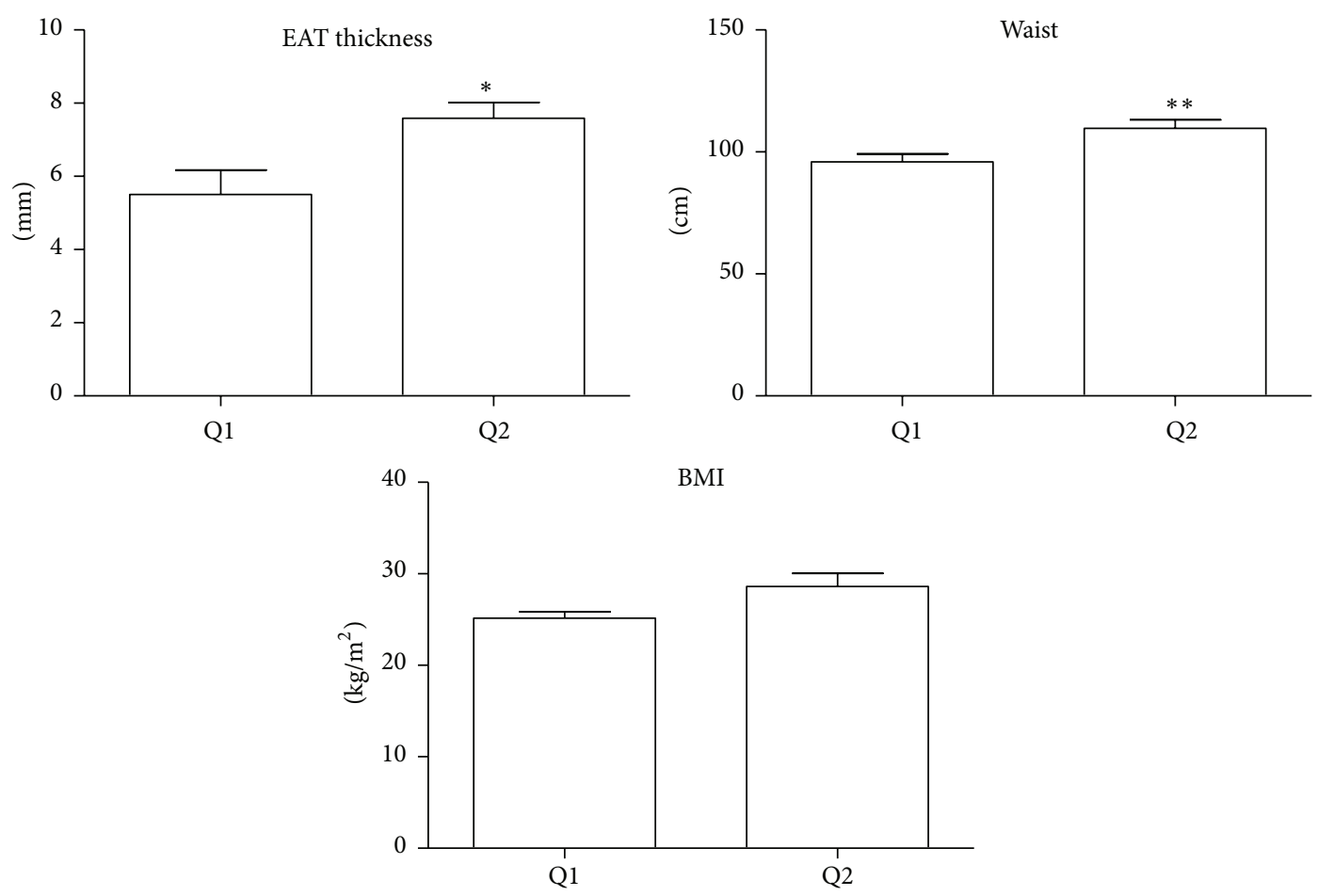

(a)
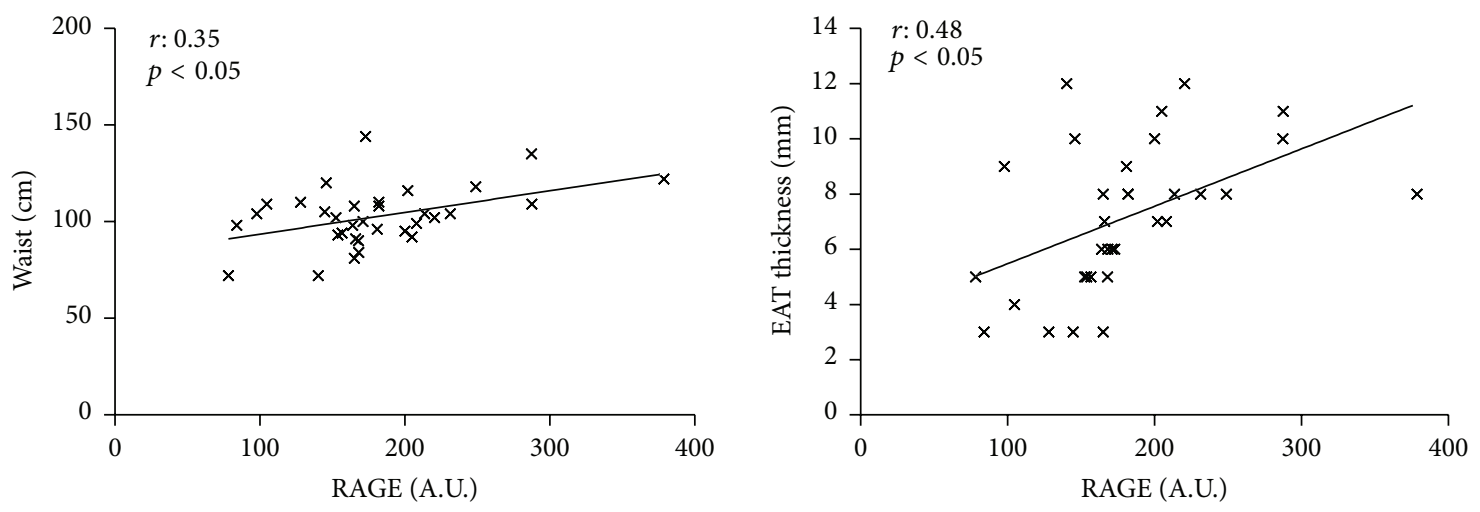

(b)

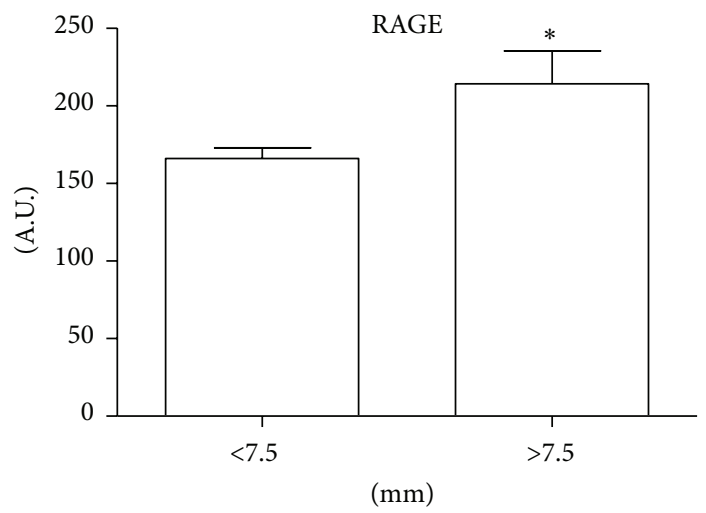

(c)

FIgURE 1: Relationship between RAGE expression in EAT, EAT thickness, and anthropometric indices in CAD patients. (a) CAD patients were stratified into two groups (Q1 and Q2) on the basis of the median RAGE expression in EAT, and EAT thickness, waist circumference, and BMI were compared in the two groups. (b) Spearman correlation analysis between mRNA RAGE level in EAT and waist circumference and EAT thickness. (c) CAD patients were stratified into two groups (Q1 and Q2) on the basis of the median EAT thickness (7.5 mm) and the levels of RAGE expression were compared in the two groups. A.U.: arbitrary unit. Data are expressed as mean $\pm \mathrm{SD} ;{ }^{*} p<0.05,{ }^{* *} p<0.01$. 

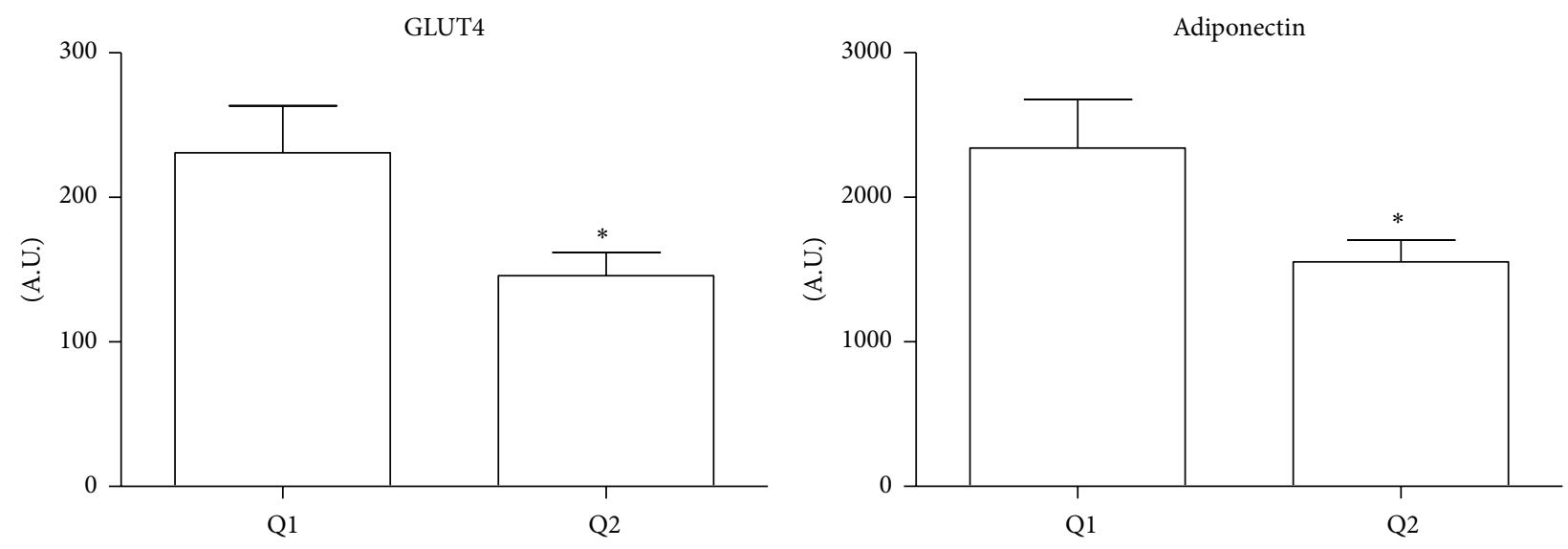

(a)
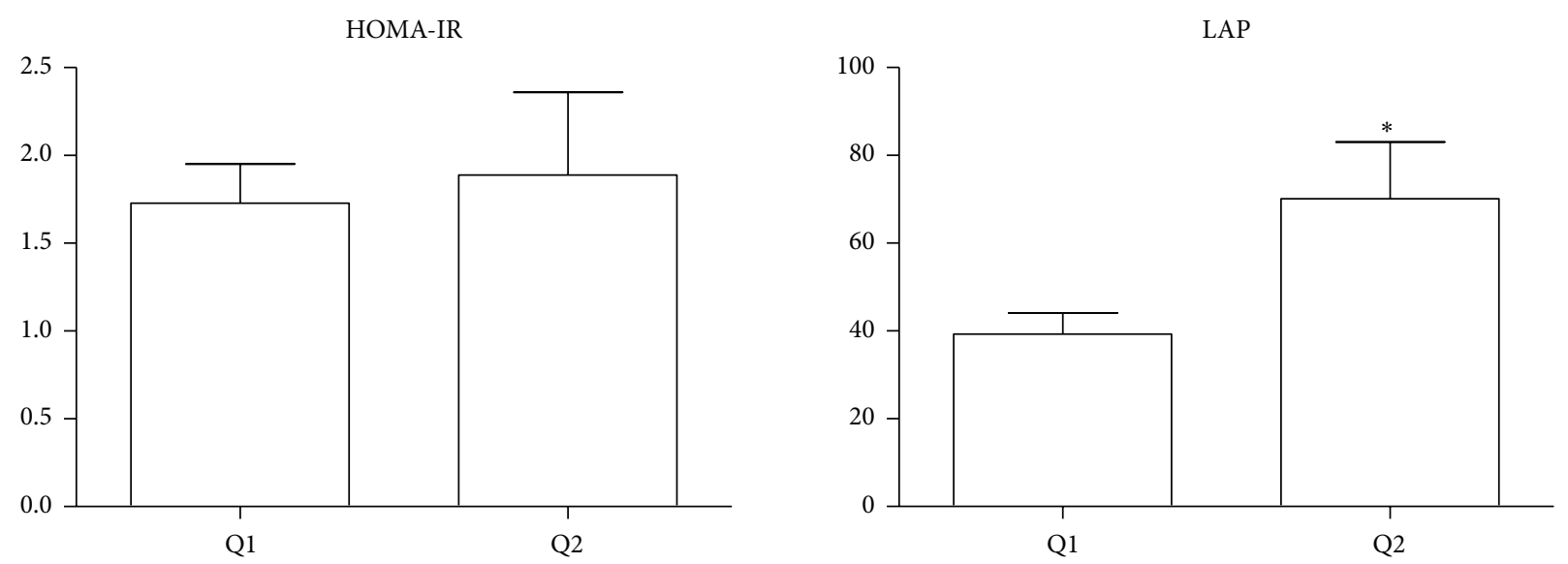

(b)

FIGURE 2: Relationship between RAGE expression in EAT and indices of insulin sensitivity. CAD patients were stratified into two groups (Q1 and Q2) on the basis of the median RAGE expression in EAT. (a) Gene expression of GLUT4 and adiponectin in EAT was compared in the two groups. (b) HOMA-IR and LAP were compared in the two groups. Data are expressed as mean \pm SD; ${ }^{*} p<0.05$.

important in the activation of the innate immune system, were higher in group Q2 (about 1.4 times, $p<0.05$ for both) (Figure 3(b)).

Correlation analyses indicated an inverse correlation between RAGE and GLO1 $(r=-0.65, p<0.0001)$ and a positive association between RAGE-TLR-4 $(r=0.51, p<0.001)$ and RAGE-MyD88 $(r=0.48, p<0.01)$.

\section{Discussion}

To the best of our knowledge this is the first human study exploring the existence of an association between the expression of RAGE in EAT, EAT metabolic dysfunctions, and adiposity in CAD patients. The findings indicate that EAT thickness as well as local tissue inflammation and insulin sensitivity seems related to local expression of RAGE.

Previous in vitro and animal studies suggested that RAGE could be involved in the progression of obesity, with a direct role in promoting adipocyte hypertrophy [7]. RAGE -/- mice at 20 weeks of age had lower weight and lower epididymal adipose tissue weight and adipocyte size than wild type mice
[7]. The observation that adenoviral RAGE overexpression in 3T3-L1 adipocytes markedly induced a hypertrophic phenotype, which was suppressed by RAGE silencing, also supports a role for RAGE in promoting adipocyte hypertrophy [7].

Our data seem to confirm that RAGE is involved in adiposity, mainly visceral, in humans too. In fact, with increasing local expression of RAGE, we observed increases in the thickness of EAT, an acknowledged visceral fat, and in WC, which serves as a marker of visceral fat accumulation. Whether this RAGE-related EAT expansion involved adipocyte hypertrophy needs to be explored further. In fact, we noted a hypertrophic state associated with the increased RAGE expression (data not shown), but since the number of patients with enough tissue for this analysis was limited, these results can only be considered preliminary.

In this study we did not directly explore the mechanisms promoting RAGE upregulation, but on the basis of previous data also from our group it would appear that both the increased inflammatory state described in EAT in CAD patients and the greater local accumulation of AGE products in expanding adipose tissue may promote this $[9,12,15-17]$. 
GLO1

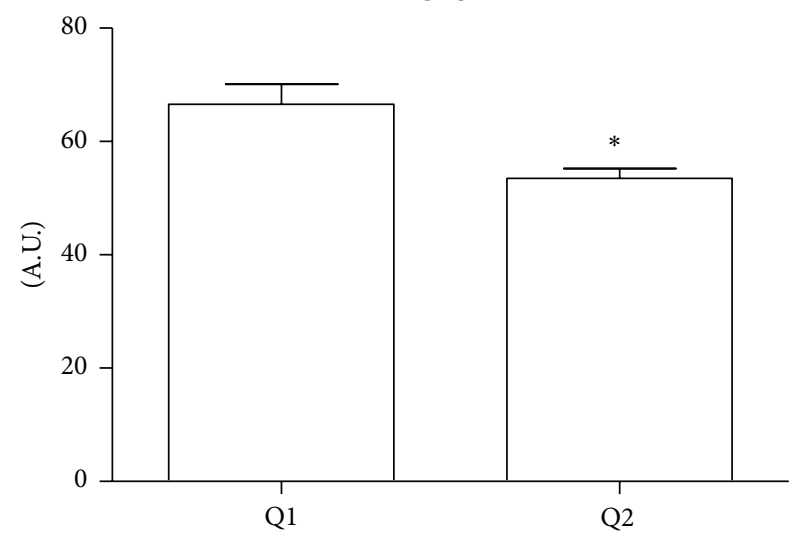

(a)

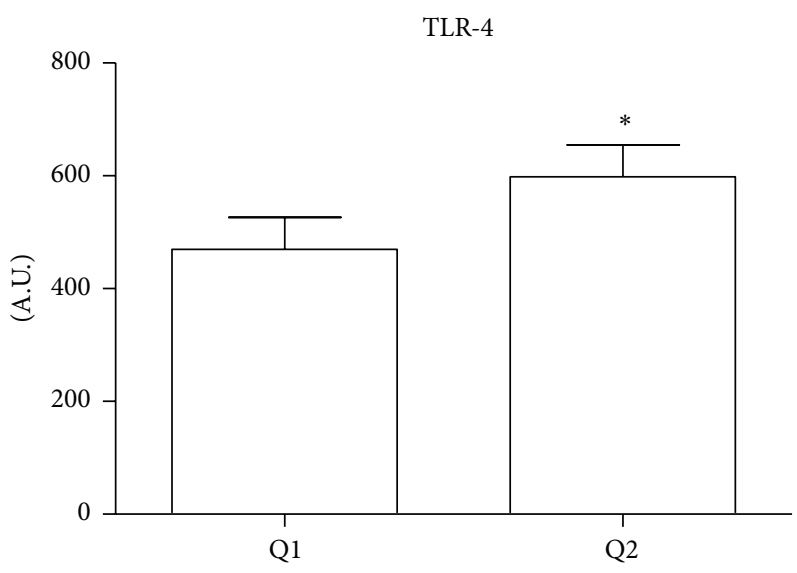

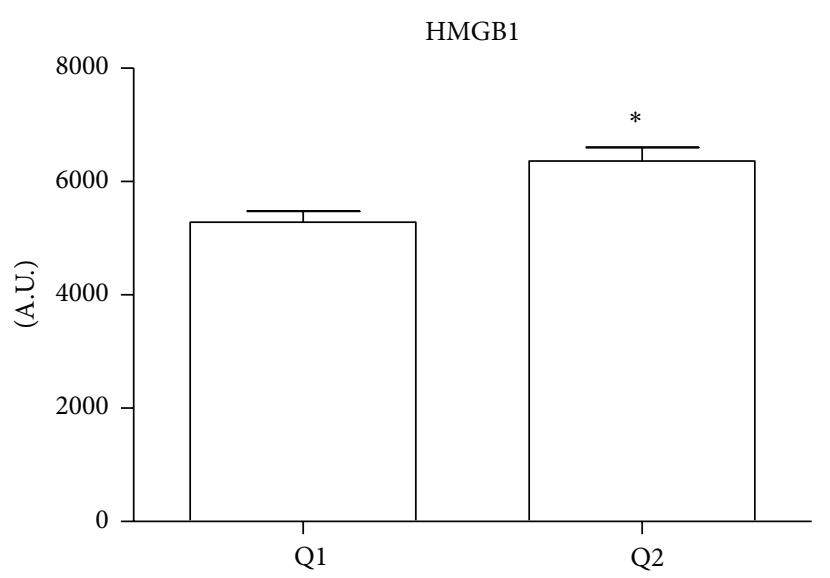

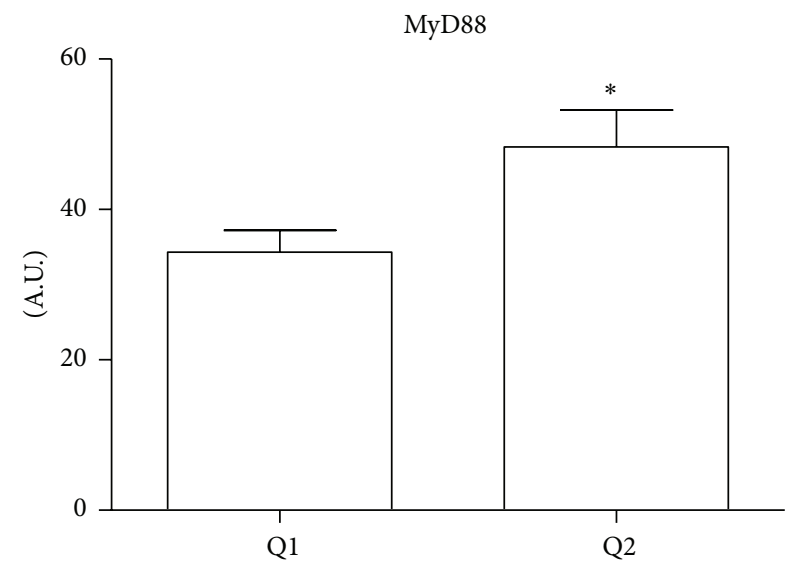

(b)

FIGURE 3: Relationship between RAGE expression in EAT and EAT inflammation. CAD patients were stratified into two groups (Q1 and Q2) on the basis of the median RAGE expression in EAT. (a) Gene expression of GLO1 and HMGB1 in EAT was compared in the two groups; (b) TLR-4 and MyD88 levels were compared in the two groups. A.U.: arbitrary unit. Data are expressed as mean \pm SD; ${ }^{*} p<0.05$.

The increase in the local production of damaging agents and reduced protection against them is also borne out by the marked reduction in GLO1, the major detoxification enzyme that protects against AGE [18], and the higher expression of HMGB1, an endogenous mediator of inflammation able to bind RAGE, promoting its expression and amplifying the inflammatory response also through activation of tolllike receptor (TLR)/MyD88 pathways. It has recently been suggested that RAGE not only shares several common ligands with the TLRs, such as HMGB1, but may also interact with MyD88, an important intracellular adaptor protein used by these receptors [19]. There is increasing evidence, therefore, of their potential synergism in amplifying inflammatory responses and our findings too suggest a link between TLR4/MyD88 and RAGE hyperexpression.

Our data also confirm that some important metabolic dysfunctions of EAT in patients with CAD may be related to RAGE overexpression. Monden et al. [7] indicated that RAGE overexpression reduced the genes involved in insulin sensitivity, such as GLUT4 and adiponectin, and attenuated insulin function. We too saw lower levels of both GLUT4 and adiponectin, with increased RAGE expression in EAT. This suggests a potential impairment of local insulin signaling. We examined insulin sensitivity in our CAD patients using the HOMA-IR, a marker of insulin resistance mainly in the liver, and LAP, a continuous variable based on WC and triglyceride concentration, two parameters which reflect tissue lipid accumulation and denote visceral adiposity $[20,21]$. Our observation that RAGE expression in EAT was mainly related to LAP reinforced the idea of a strong correlation between local RAGE overexpression, fat accumulation, and impaired insulin sensitivity.

Our study has some limitations. The first one is the lack of data on protein expression. Since isolation of EAT during surgery is a delicate and difficult procedure and the amount of tissue isolated is often poor and not enough to perform both gene and protein expression analyses, in this study we first decided to carry out a gene expression study. The lack of quantification of local AGE as well as the evaluation of other molecules, which may promote RAGE upregulation, may also represent a second important limit. The third limitation is that we included only males, so presently we cannot check 
for possible gender-related differences. Only through new patient enrollment, of both sexes, we will be able to perform protein expression quantification to study which specific pathways/molecules drive RAGE expression in EAT as well as clarify the existence of potential gender-related differences. Finally, the comparison between EAT and other kinds of fat depots, not performed in this study, could be helpful to reinforce our data on the role of RAGE in linking EAT metabolic dysfunction and CAD.

\section{Conclusions}

In conclusion this study's findings suggest the potential involvement of RAGE in promoting EAT dysfunction in CAD patients. Whether RAGE could also be a potential target to reduce EAT-induced cardiovascular and other complications needs to be explored further.

\section{Conflict of Interests}

The authors declare that they have no conflict of interests regarding the publication of this paper.

\section{Authors' Contribution}

Elena Dozio, Lorenza Tacchini, and Massimiliano Marco Corsi Romanelli designed the study. Elena Dozio, Elena Vianello, and Silvia Briganti acquired and analyzed data. Elena Dozio wrote the paper. John Lamont, Lorenza Tacchini, Gerd Schmitz, and Massimiliano Marco Corsi Romanelli critically revised the paper.

\section{Acknowledgments}

The authors thank Dr. G. Cassetti and Dr. L. Menicanti, I.R.C.C.S. Policlinico San Donato, for patient enrollment and EAT isolation; Dr. F. Bandera, I.R.C.C.S. Policlinico San Donato, for quantifying EAT thickness by echocardiography; Dr. T. Konovalova and Dr. A. Sigruener, University of Regensburg, for bioinformatic support and microarray assays; and Dr. E. Costa, I.R.C.C.S. Policlinico San Donato, for clinical chemistry data. The study was supported by funds from the Italian Ministry for Health "Ricerca Corrente" IRCCS Policlinico San Donato, internal funds from the University of Regensburg, and the EU Framework 7 project "LipidomicNet."

\section{References}

[1] G. Iacobellis, A. E. Malavazos, and M. M. Corsi, "Epicardial fat: from the biomolecular aspects to the clinical practice," International Journal of Biochemistry and Cell Biology, vol. 43, no. 12, pp. 1651-1654, 2011.

[2] T. Chavakis, A. Bierhaus, N. Al-Fakhri et al., "The pattern recognition receptor (RAGE) is a counterreceptor for leukocyte integrins: a novel pathway for inflammatory cell recruitment," Journal of Experimental Medicine, vol. 198, no. 10, pp. 1507-1515, 2003.
[3] Y. Yamamoto, I. Kato, T. Doi et al., "Development and prevention of advanced diabetic nephropathy in RAGE-overexpressing mice," The Journal of Clinical Investigation, vol. 108, no. 2, pp. 261-268, 2001.

[4] A. Soro-Paavonen, A. M. D. Watson, J. Li et al., "Receptor for advanced glycation end products (RAGE) deficiency attenuates the development of atherosclerosis in diabetes," Diabetes, vol. 57, no. 9, pp. 2461-2469, 2008.

[5] H. Unoki, H. Bujo, S.-I. Yamagishi, M. Takeuchi, T. Imaizumi, and Y. Saito, "Advanced glycation end products attenuate cellular insulin sensitivity by increasing the generation of intracellular reactive oxygen species in adipocytes," Diabetes Research and Clinical Practice, vol. 76, no. 2, pp. 236-244, 2007.

[6] A. Z. Kalea, A. M. Schmidt, and B. I. Hudson, "RAGE: a novel biological and genetic marker for vascular disease," Clinical Science, vol. 116, no. 8, pp. 621-637, 2009.

[7] M. Monden, H. Koyama, Y. Otsuka et al., "Receptor for advanced glycation end products regulates adipocyte hypertrophy and insulin sensitivity in mice: involvement of toll-like receptor 2," Diabetes, vol. 62, no. 2, pp. 478-489, 2013.

[8] K. H. J. Gaens, G. H. Goossens, P. M. Niessen et al., "Nc(carboxymethyl)lysine-receptor for advanced glycation end product axis is a key modulator of obesity-induced dysregulation of adipokine expression and insulin resistance," Arteriosclerosis, Thrombosis, and Vascular Biology, vol. 34, no. 6, pp. 11991208, 2014.

[9] T. Mazurek, L. Zhang, A. Zalewski et al., "Human epicardial adipose tissue is a source of inflammatory mediators," Circulation, vol. 108, no. 20, pp. 2460-2466, 2003.

[10] P. Iozzo, "Myocardial, perivascular, and epicardial fat," Diabetes Care, vol. 34, no. 2, pp. S371-S379, 2011.

[11] A. E. Malavazos, M. M. Corsi, F. Ermetici et al., "Proinflammatory cytokines and cardiac abnormalities in uncomplicated obesity: relationship with abdominal fat deposition," Nutrition, Metabolism and Cardiovascular Diseases, vol. 17, no. 4, pp. 294302, 2007.

[12] E. Dozio, G. Dogliotti, A. E. Malavazos et al., "IL-18 level in patients undergoing coronary artery bypass grafting surgery or valve replacement: which link with epicardial fat depot?" International Journal of Immunopathology and Pharmacology, vol. 25, no. 4, pp. 1011-1020, 2012.

[13] G. Iacobellis, F. Assael, M. C. Ribaudo et al., "Epicardial fat from echocardiography: a new method for visceral adipose tissue prediction," Obesity Research, vol. 11, no. 2, pp. 304-310, 2003.

[14] V. G. Tusher, R. Tibshirani, and G. Chu, "Significance analysis of microarrays applied to the ionizing radiation response," Proceedings of the National Academy of Sciences of the United States of America, vol. 98, no. 9, pp. 5116-5121, 2001.

[15] X. Jia, T. Chang, T. W. Wilson, and L. Wu, "Methylglyoxal mediates adipocyte proliferation by increasing phosphorylation of Akt1," PLoS ONE, vol. 7, no. 5, Article ID e36610, pp. 1-9, 2012.

[16] E. Dozio, A. E. Malavazos, E. Vianello et al., "Interleukin-15 and soluble interleukin-15 receptor alpha in coronary artery disease patients: association with epicardial fat and indices of adipose tissue distribution," PLoS ONE, vol. 9, no. 3, Article ID e90960, 10 pages, 2014.

[17] E. Dozio, S. Briganti, E. Vianello et al., "Epicardial adipose tissue inflammation is related to vitamin D deficiency in patients affected by coronary artery disease," Nutrition, Metabolism and Cardiovascular Diseases, vol. 25, no. 3, pp. 267-273, 2015. 
[18] N. Rabbani and P. J. Thornalley, "Methylglyoxal, glyoxalase 1 and the dicarbonyl proteome," Amino Acids, vol. 42, no. 4, pp. 11331142, 2012.

[19] H. S. Hreggvidsdottir, T. Östberg, H. Wähämaa et al., "The alarmin HMGB1 acts in synergy with endogenous and exogenous danger signals to promote inflammation," Journal of Leukocyte Biology, vol. 86, no. 3, pp. 655-662, 2009.

[20] D. S. Ludwig, "The glycemic index: physiological mechanisms relating to obesity, diabetes, and cardiovascular disease," The Journal of the American Medical Association, vol. 287, no. 18, pp. 2414-2423, 2002.

[21] H. S. Kahn, “The 'lipid accumulation product' performs better than the body mass index for recognizing cardiovascular risk: a population-based comparison," BMC Cardiovascular Disorders, vol. 5, article 26, pp. 1-10, 2005. 


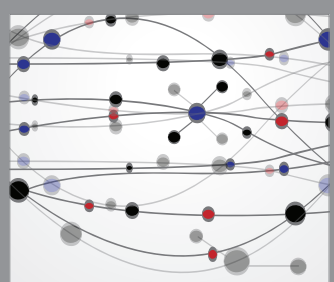

The Scientific World Journal
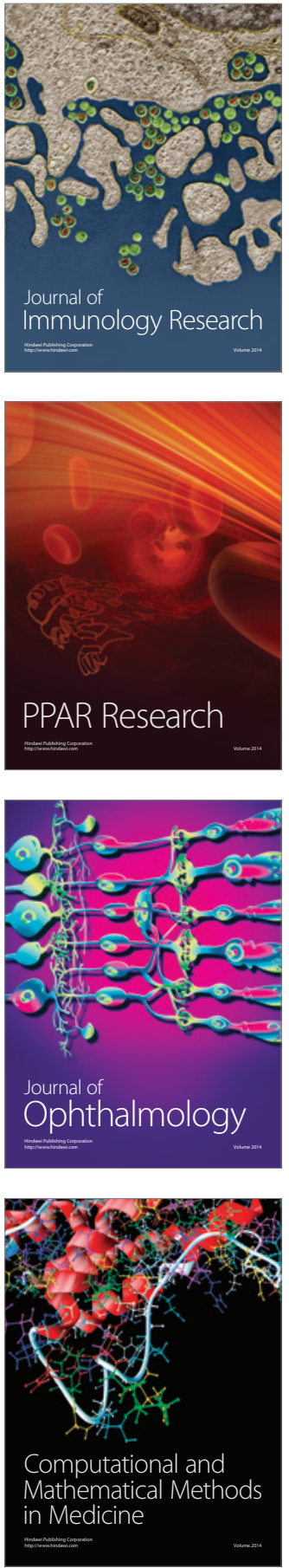

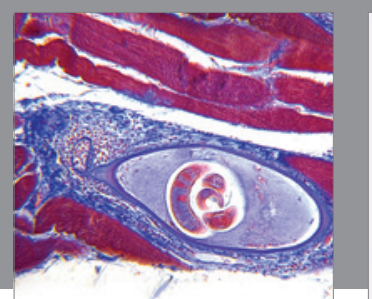

Gastroenterology Research and Practice

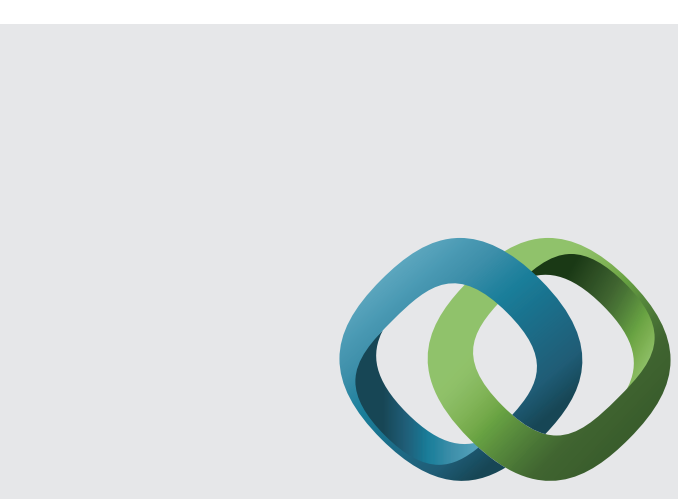

\section{Hindawi}

Submit your manuscripts at

http://www.hindawi.com
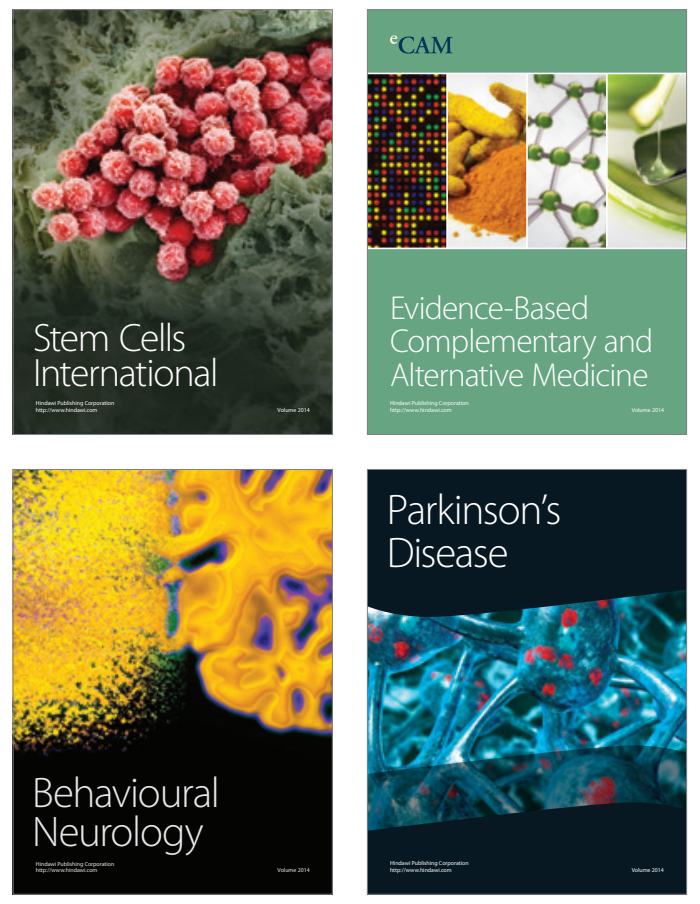
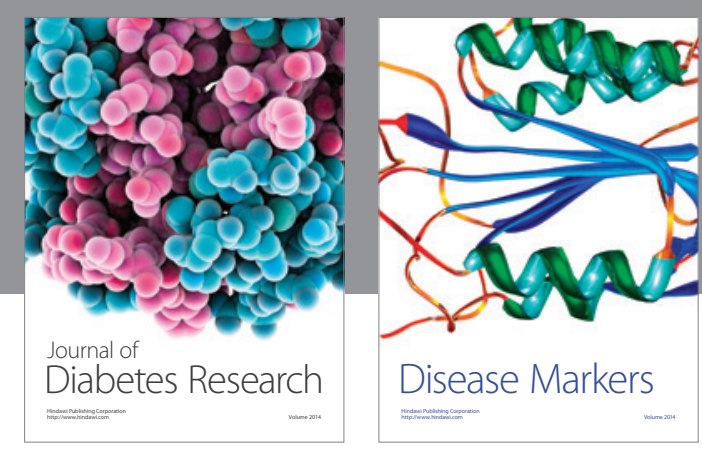

Disease Markers
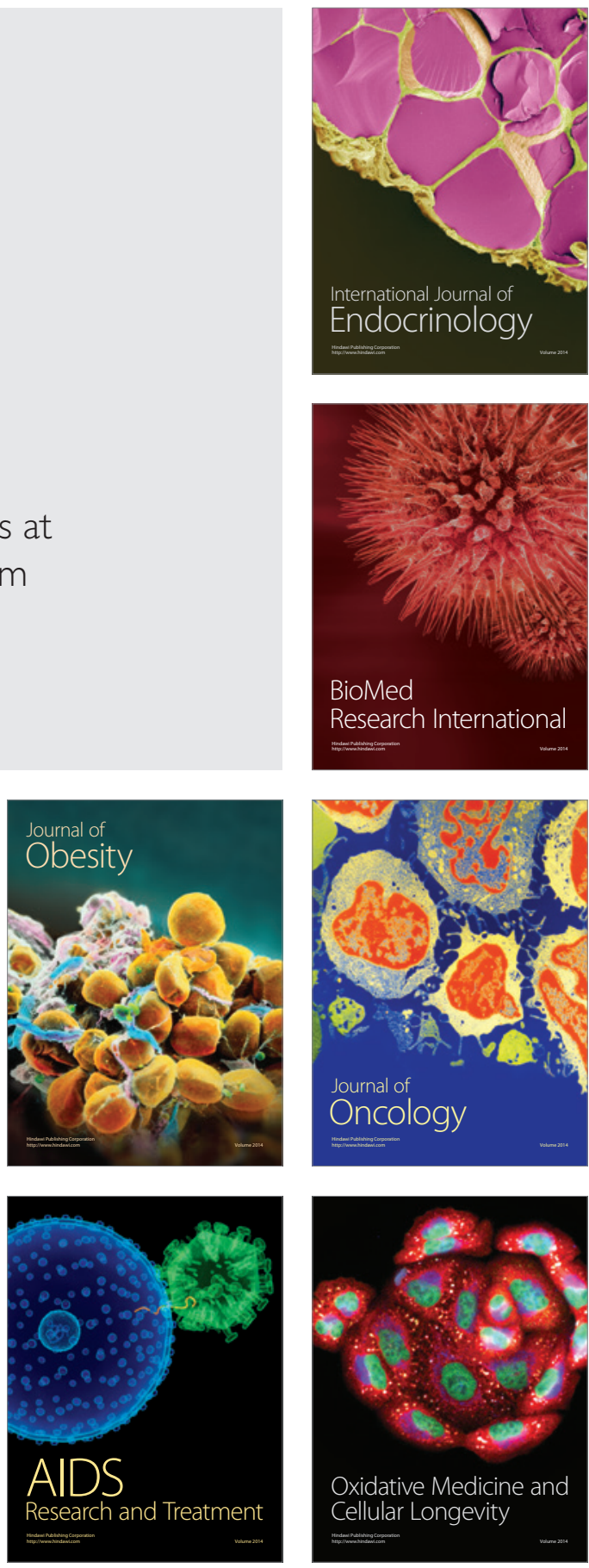